\title{
Stability of material in dynamics of structural organization
}

\author{
Valery Vyrovoy ${ }^{1}$, Vladimir Sukhanov ${ }^{1}$, and Oksana Korobko ${ }^{2, *}$ \\ ${ }^{1}$ Odessa State Academy of Civil Engineering and Architecture, Department of Production of Building \\ Products and Constructions, Didrihsona st. 4, 65029 Odessa, Ukraine \\ ${ }^{2}$ Odessa State Academy of Civil Engineering and Architecture, Department of Architectural \\ Structures, Restoration and Reconstruction of Buildings, Constructions and their Complexes, \\ Didrihsona st. 4, 65029 Odessa, Ukraine
}

\begin{abstract}
Formation of the scientific ideology is based on the basic model of the structured medium and demands the formalization of the term «structure». The objects-systems are offered to be present in the form of models. It is shown that the mechanism of structure formation on the solid level where gravity prevails differs from mechanism of structure formation at the level where forces of interparticle interactions dominate. It is asserted that geometrical characteristics of products define conditions of the organization of its integral structure. Rather independent coexistence of various solid levels assumes spontaneous realization of various interconnected events which provides the wholeness and safe functioning of a construction-system.
\end{abstract}

\section{Introduction}

The content of every paradigm consists of the system of scientific views based on the general model representations defined the entire set of conceptual, methodological, theoretical and experimental regulations [1, 2]. According to I. Prigozhin [3] such a system of natural scientific views forms scientific ideology. He noticed "...the science is an ideology, it is also rooted in the culture" [p. 49, 3]. According to the scientific ideology, a supporter of which I. Prigozhin was, one can argue that with the growing of internal conflicts inside the dominant scientific views the movement trajectory of this paradigm ideas gets in the bifurcation zone. It leads to the shift of paradigm and thereby it causes the displacement of scientific ideologies. A change of ideologies including scientific ones is a lengthy process often painful and tragic. It must be taken into account that it is talked not about replacement "bad by good", "backward by progressive" etc. Drifting or change of paradigm implies the formation of a new system of views based on fundamentally different basic models.

Transition through the point of bifurcation changes the paradigm structure defining its content meaningfulness, the level of its methodological provision, theoretical saturation. The other principles are being formed and scientific schools are being created to promote

\footnotetext{
* Corresponding author: okskorobko71@gmail.com
} 
the ideas of the new paradigm. The example is the change in the general scientific basic concepts of material (including building) science.

\section{Portraits of structure of building composites in dynamics of evolution}

Simple, clear, accepted on faith the base model of building materials as the solid medium allows inventing the methodology of their assessment on the level of the average. The explanation of building materials mechanism formation is based on the ideology of reductionism (lat. reduction - return, bringing back) that comes from the assumption that the whole can be understood just if its parts are understood. The methods of designing building products and constructions are based on the model of allowable loads and deformations, which should be below the experimentally determined limit values. On these basic principles many generations of ideologists of unstructured medium, its analysis and calculation and design methods both separate structural elements and structures, buildings and constructions with various kinds and purposes have been taught.

Ideology of unstructured medium implies an ambiguity of perception and evaluation of reality. There is the certain dualism in description of surroundings. There are originality and uniqueness of the processes and objects based on their structural dissimilarity on the one hand. There is unstructured medium on the other hand that is assessed average parameters of temperature, humidity and pressure. They differ in density and strength values, ratios of thermal conductivity, thermal expansion etc. Reality dissolves in the medium quality indicators accepted in the ideology of unstructured medium.

Emerged dualism can be considered as the beginning of the inner conflict within current paradigm. The system approach and synergy in its successful development arise from particular structure existing in objects of different nature and use. The holism ideology is forming (gr. holos - the whole, entire), the main point of it was declared by Aristotle (384 $322 \mathrm{BC})$. He stated that the whole includes more than is contained in the simple sum of its constituent parts. The emergence and expression of something new is connected with the structural organization of objects-systems. The key parameter for creating the unexpected one is a structure. Accepting a structure as existing phenomena one can conclude that the ideological scientific positions start to change. Nicolay Gumelyov's words are appropriate in this case "the whole world is discovered again for me". The belief system ideologically oriented to the structure accountability for expressing and maintaining the properties of any object is forming.

In the evolution of different approaches and views a problem of terminology arises. Generally at the beginning existing terminology is used and some additional sense is given. Such a type of term that is widely used in different fields of knowledge is a term structure.

Widespread use of any term spreads its meaning to the blurriness. It is logically to say "language structure", "society structure", "metal structure", "concrete structure" and other ones. The term is used appropriately in biology, medicine, sociology, geology, mechanics, physics, chemistry, materials science and other sciences. The combined principal for objects not similar to their nature is certain hierarchy of their inner structure, inner architecture existed in the synthetic term structure.

Generally the structure (lat. structura - arrangement, order) is understood as a set of stable relations of object satisfied its integrity and identity to itself. In another word it is preserving the basic properties at various external and internal changes [4]. This definition assumes that the structure reflects some fixed condition of the object, which allows qualitatively and quantitatively evaluating and describing its structure taking into account the interaction and the relative position of the totality of the structural elements and groups of them. According to [5] structure can be considered as "stopped motion" or as moment 
fixed condition of the system in the process of its development, as certain configuration of its components. It is worth to say that term "structure" is used successfully in the description of dissipative systems [6], cooperative phenomena [7], processes of selforganization and adaptation various systems [8] without accurate definition of the essential content. This was the basis to bring into the essential content certain dynamics and selfsupport in autopoietic system [9], systems whose development goes with an exacerbation [10]. Quite a lot of individual definitions of the term "structure" in separate crystal, metal, polymer and other material descriptions are suggested. Multiplicity of definitions of the same concept assumes its inconsistency to the generalized content as the standard term. Therefore, it seems logical to limit directly the semantic meaning of the term by providing structure in the form of a specific model (lat. modulus - measure, sample) of the object [11]. This method allows focusing on the structural features of the object which are responsible for the manifestation of certain properties according to a specific subject. Choice of model structure depends on the subjective position of a particular investigator his ideological positions, research objectives, methodology, instrumental and material resources, etc. The subjective nature of the "appointment" of the structure dominant elements is contained in the method of expert evaluations that basically allows estimating prevailing ideology of community of professionals in a time interval. Therefore making a particular model the purpose of the structural description of a particular object (the mechanism, the process) must be defined. One of the purposes of the structural approach is identifying the structural controllable factors which allow the structure to reproduce a required set of structural elements. To do this fixing a large-scale description of the boundaries and establishing a number of priority structure elements are needed to ensure the purpose of the object of analysis.

A many studies are devoted to allocation the scale levels on the size principle in the description of the structure of different nature materials (e.g. [12, 13]). There are supposed to allocate the levels of structural inhomogeneities on the basis of similarity of structural organization mechanisms in the publications $[11,14,15]$ that allows to descript a product or constructional integral structure presented in the form of a system of a certain kind [16]. In the process of analyzing the structure organizational mechanism the borders of scales were found taking into account the principal difference in the processes implemented under the influence of force of gravity from the processes that almost do not react to the forces of gravity. V.I. Vernadskiy [17] noticed that macroscopic niche exists where a worldwide attraction reigns. He emphasized that a spatial multiscalement associates with timing multiscalement. This position is similar to H. Bergson's statement [18] that multiple of levels of time and a multiplicity of life worlds exist at different levels. It is pertinently from our point of view to mention E. Petitot's [19] conclusions about emergencity (eng. emergence - appearance of a new one) existence and display two (at least) different levels of organization are required - one is underlying "micro" and another phenomenal "macro" observed at various spatial and temporal scales. The compulsory presence of a time series is noteworthy when the complex behavior of complex systems is considered [20, 21, 22]. A priori we will proceed from the time invariance (lat. invariance - unchanging) at different spatial scale levels without analyzing the nature of time. Within certain time slot (time quantum) the number of taking place and implemented events are fundamentally different, up to impalpability and therefore insensitivity [20].

The principal scheme of different scale structure coexistence is shown in Fig. 1.

The certain scale level, involved in the level of metastable state (MS) where individual particles and their families can reside in a metastable state, is allocated in a separate level. In the case of disintegration of the unit or particles cleavage from it (caused by physic and chemical, physical and physical and mechanical processes) the last transfer at the level where gravity is not dominant. When particles are combined into structural aggregates 
which weight is higher than force interactions of particles aggregate they fall under the effect of gravity automatically. It can be supposed that MS-level is a link (horizon of events) between two spatial levels. These levels differ in the causes of the occurrence and in the quantity of certain events occurring during a certain time quantum.

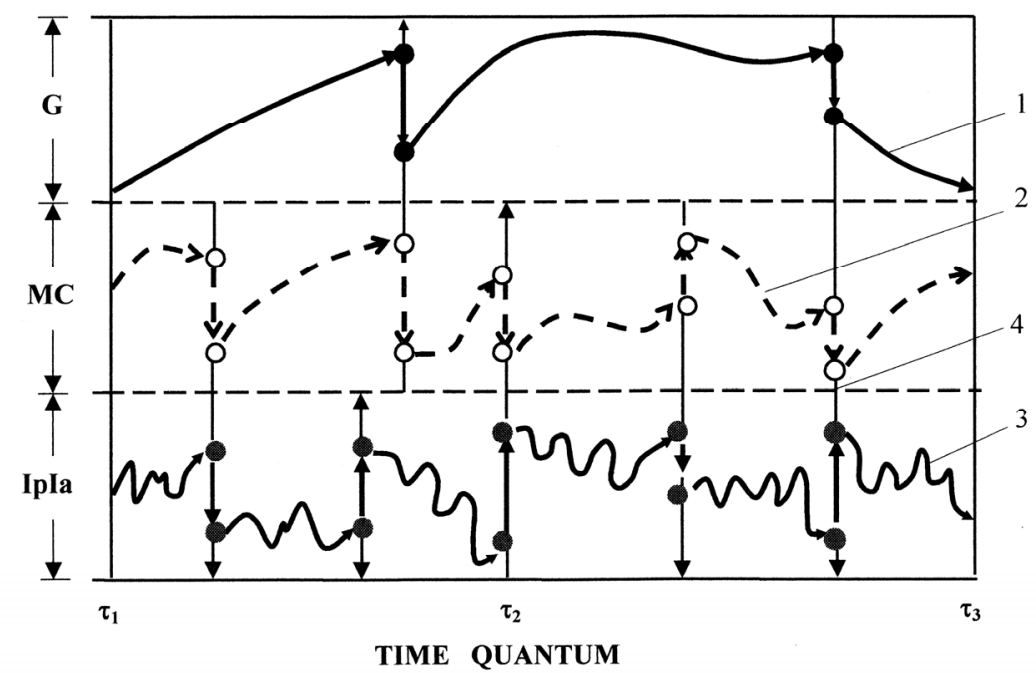

Fig. 1. Diagram of multiscaled interactions. $\mathrm{G}$ - scale level where gravity proceeds; $\mathrm{MC}$ - scale level where gravity and interparticle interactions can proceed; IpIa - scale level where interparticle interactions proceed; $1,2,3$ - events at multi- scale levels of structure; 4 -interactions between the structural components at multi-scale levels.

The difference between the mechanisms of structure formation at various spatial levels and the implementation of these acts of structure organization basically not matching in time suggest a certain level of independent existence. Processes occurring at every level subject to the laws manifested at this level, and cannot be carried out at another scale level in most cases. Impact of an event from one level on occurrence of events at another one relates to the rank of external events and occurs usually in mismatched time slots. It means certain autonomy of developments at every level and a certain coexistence of diverse events in the whole system. It can be argued that this coexistence, realized through interaction of diverse events, provides the appearance of new (the other) qualitative characteristics of each scale level and the system as a whole.

Here the events are understood as a set of processes and phenomena of different nature implemented at different spatial scales for different time quantum. The problem arises to associate flows of events related but fundamentally different in essence occurring at different spatial scales and during different time lengths. It needs to identify elements that can be present at all scale levels simultaneously or belong to a specific spatial level and which are capable to provoke the birth of a certain event by their presence, Fig. 1.

In order to determine these elements the task is to trace the formation history of the structure of a particular object. In publications $[11,14,15]$ it is underlined that geometric characteristics of a particular product have a significant impact on the structure which components are local and integral field of residual (primary, technological, hereditary) deformations. It allows concluding that directed organization structure with a set of structural elements arises from the impact of the individual characteristics of a particular product. Geometric image of a specific object creates unique qualitative and quantitative ratios of the structural components at different spatial levels with temporal difference of 
initiation of events displaying. The geometrical parameters of objects should be regarded as significant management factors of the initial structure organization becoming the objectproduct. Thus the developed model of the structure should be related to a particular object (products, structures, etc.).

\section{Functional purpose of structural elements}

Structure models of a particular object should contain elements aimed at ensuring sustainable relationships to ensure the integrity and identity of the object under the action of all operational loads. An earlier analysis [14] allowed us to offer a classification of structural elements based on the difference in the speed of response of various elements when object is subjected to external and internal factors. In general case conservative metastable and active elements are marked.

Conservative (lat. conservation - saving) structural elements include elements changed their parameters slow enough during period when object functions. Usually conservative elements belong to the large-scale level where dominant force is gravity and phenomenological approaches of properties evaluation are implemented.

Elements of the structure which are relatively stable under these conditions and able to spontaneously in the stable or unstable state by the action of external or internal factors are referred to the metastable (gr. meta - between, after, through) elements of the structure.

The hallmark of active (lat. activus - activities) structural elements is their ability to respond adequately to the proven impact in a proportion time interval (one tempo rhythm).

Under the structure elements (lat. elementum - element, the original substance) various scale components of the system with specific features ensuring certain functions that contribute to the objectives of each structure level and the system as a whole are suggested for understanding. Structure elements may be specific material formation (e.g., components of the system, the pores and capillaries, cracks, etc.) of the system defining its internal state (e.g., local and integral technological and operational strain, osmotic phenomena, etc.).

System gets the entire set of structural elements in the form of construction products and structures [14] in the process of concrete receiving and its processing into products. At this stage, all the original members are actively involved in multiscale structural design with the formation of new structural elements of the becoming system. The situations are not excluded where creating intermediate structural formations initiate the formation of the intermediate structural birth and development of fundamentally new elements included in the structure of the final product. Equifinality effects are characteristic (lat. aequus - equal proportionate; finalis - final). In our case it means the implementation of the structural organization of the various mechanisms that lead to the emergence of qualitatively identical structure elements. As examples, the origin of technological cracks is considered: - in the formation of discrete structures at the product level and at the level of tumors interaction of initial grain mineral binders; - in the development of gradients of own strains at the interfacial hardening matrix material and fillers; - under the influence of emerging gradient deformation at the level of product structures $[11,14]$.

A kind of "albums portraits" multiscale structures coexisting on different time scales are created as a result of mutual influence and interaction of all elements at all levels of irregularities. "Albums of portraits" means the permanent dynamics of structural components the totality of which defines a "family portrait" of the structure within a fixed time interval. In this context, a comparison with a "family portrait" suggests that at the same time it has all the elements of the family system. Thus the structure-system comes into the active phase of operation with a set of conservative, metastable and active elements of the structure. Further safe operation of the structure-system depends on the ability of the structural elements by self-consistent interactions and by transforming their own parameters 
to ensure the preservation of the system properties within continuous operational loads exposure.

Ensuring the stability of the properties for the required time of operation raises the problem of exception and analysis of models and structures in which adaptation processes (lat. adaptation - adjustment) can be implemented by percolation phenomena of selforganization at all various scales and multi time levels of structure-system. Adaptation of concretes $[23,24]$ should be attributed to the underlying determinants of the viability of production systems. Therefore a number of priority elements that can alter the periods of adverse events should be defined in the model of the structure. This will identify the main governing factors aimed at creating a "family portrait" of the construction-system structure operated under adverse external influences.

\section{Conclusions}

A structured approach at this stage of its development should be considered as "an introduction to the explanation" of the mechanisms and processes of creation and functioning of systems of various types and purposes. It is based on the becoming scientific ideology based on the ideas and methods of a systematic approach and synergy for which the term "structure" is the dominant concept. There is a paradigms shift based on the basic model of continuous medium in the direction of paradigms based on models of a structured self-developing medium. The fulfilled analysis has led to the conclusion that geometrical characteristics are one of the factors influencing the mechanisms of formation of building products and construction. Therefore, studying the structure of the material without its registration in some constructive forms is futile. In addition, due to blurring and information uncertainty of the generalized definition of the term "structure" it is proposed to develop and analyze models of the structure of concrete products and constructions. In this case, it is necessary to take into account the influence of spatial different-scale levels on the mechanisms of interdependent structural organization of each level of the integrated structure of the construction-system at non-simultaneous realization certain events during the fixed quantum of time. This allows us to consider the structure in a certain dynamics of development both in the establishment of the system and the action of its duty operation. It is proposed to classify the elements of the structure by the time of their response to external and internal influences on the conservative metastable and active elements of individual subsystems and the system itself.

\section{References}

1. T. S. Kuhn, The Structure of Scientific Revolutions (AST MOSCOW, Moscow, 2009)

2. Br. Latour, Science in Action: How to follow scientists and engineers through society (European University in St. Petersburg Press, St. Petersburg, 2013)

3. I. Prigogine, The philosophy of instability, J. Problems of Philosophy, 6, 45-57 (1991)

4. Soviet Encyclopedic Dictionary (Soviet Encyclopedia, Moscow, 1980)

5. E. Soroko, Golden ratios, processes of self-organization and evolution of systems: Introduction to the general theory of harmony systems (KomKniga, Moscow, 2006)

6. G., Nicolis, I. Prigogine, Exploring Complexity: An Introduction (LCI, Moscow, 2008)

7. H. Haken, Information and Self-Organization. A Macroscopic Approach to Complex Systems (Mir, Moscow, 1991)

8. Synergetics: Research and technologies (Book House "LIBROKOM", Moscow, 2009) 
9. H. R. Maturana, F. J. Varela, The Tree of Knowledge: The Biological Roots of Human Understanding (Progress-Traditsija, Moscow, 2001)

10. E. Knyazeva, S. Kurdyumov, Grounds of Synergetics. Synergetic Vision of the World (KomKniga, Moscow, 2005)

11. V. Solomatov, V. Vyrovoy, V. Dorofeev, A. Sirenko, Composite building materials and constructions of the reduced material capacity (Budivelnik Press, Kiev, 1991)

12. I. Rybev, Building materials on the basis of binders (Vysshaja shkola Press, Moscow, 1978)

13. A. Sheikin, The structure, strength and crack resistance of a cement stone (Strojizdat Press, Moscow, 1974)

14. V. Vyrovoy, V. Dorofeev, V. Sukhanov, Composite building materials and constructions. Structure, self-organization, properties (TES Press, Odessa, 2010)

15. S. B. Fic, V. Vyrovoy, V. Dorofeev, The processes of self-organization structure of composite building materials (Politechnika Lublenska Press, Lublin, 2013)

16. V. Vyrovoy, V. Dorofeev, V. Sukhanov, J. Econ. Res. mater., struct., build., constr. 41, 133-139 (2008)

17. V. Vernadsky, Philosophical thoughts of the naturalist (Nauka, Moscow, 1998)

18. J. Bergson, Creative evolution. Matter and memory (Harvest, Minsk, 1999)

19. E. A. Petito, Semiofizika and metaphysical realism. Synergetics. Anthology. 1, 329342 (2013)

20. A. Alyushin, E. Knyazeva, Tempo-worlds: Speed of perception and time scales (Publishing House "LCI" Press, Moscow, 2008)

21. I. Prigogine, I. Stengers, Time, chaos, quantum (Progress Press, Moscow, 1994)

22. S. W. Hawkins, Three books about space and time (Amphora, St. Petersburg, 2014)

23. L. Sheynich, K. Pushkareva, The processes of self-organization of structure of build composites (Gamma-Print Press, Kiev, 2009)

24. V. Cherniavsky, Adaptation abiotic systems: concrete and reinforced concrete (DNURT, Dnipropetrovsk, 2008) 